(c) 2020 IEEE. Personal use of this material is permitted. Permission from IEEE must be obtained for all other uses, in any current or future media, including reprinting/republishing this material for advertising or promotional purposes, creating new collective works, for resale or redistribution to servers or lists, or reuse of any copyrighted component of this work in other works.

\title{
SECRET: Semantically Enhanced Classification of Real-world Tasks
}

\author{
Ayten Ozge Akmandor, Student Member, IEEE, Jorge Ortiz, Member, IEEE, Irene Manotas, Bongjun Ko, \\ and Niraj K. Jha, Fellow, IEEE
}

\begin{abstract}
Supervised machine learning (ML) algorithms are aimed at maximizing classification performance under available energy and storage constraints. They try to map the training data to the corresponding labels while ensuring generalizability to unseen data. However, they do not integrate meaning-based relationships among labels in the decision process. On the other hand, natural language processing (NLP) algorithms emphasize the importance of semantic information. In this paper, we synthesize the complementary advantages of supervised ML and NLP algorithms into one method that we refer to as SECRET (Semantically Enhanced Classification of REal-world Tasks). SECRET performs classifications by fusing the semantic information of the labels with the available data: it combines the feature space of the supervised algorithms with the semantic space of the NLP algorithms and predicts labels based on this joint space. Experimental results indicate that, compared to traditional supervised learning, SECRET achieves up to $14.0 \%$ accuracy and $13.1 \%$ F1 score improvements. Moreover, compared to ensemble methods, SECRET achieves up to $12.7 \%$ accuracy and $13.3 \% \mathrm{~F} 1$ score improvements. This points to a new research direction for supervised classification based on incorporation of semantic information.
\end{abstract}

Index Terms-Feature space, inference, machine learning, natural language processing, semantic information, semantic space, word embedding.

\section{INTRODUCTION}

$S^{1}$ GNIFICANT progress has been made in natural language processing (NLP) and supervised machine learning (ML) algorithms over the past two decades. NLP successes include machine translation, speech/emotion/sentiment recognition, machine reading, and social media mining [1]. Hence, NLP is beginning to become widely used in realworld applications that include either speech or text. Supervised ML algorithms excel at modeling the data-label relationship while maximizing performance and minimizing energy consumption and latency.

Supervised ML algorithms train on feature-label pairs to model the application of interest and predict labels. The label involves semantic information. Palatucci et al. |2] use this information through vector representations of words to find the novel class within the dataset. Karpathy and Fei-Fei [3] generate figure captions based on the collective use of image datasets and word embeddings. Such studies indicate that data features and semantic relationships correlate well. However, current supervised ML algorithms do not utilize such correlations in the decision-making (prediction) process. Their decisions are only based on the feature-label relationship, while neglecting significant information hidden in the labels, i.e., meaning-based (semantic) relationships

This work was supported by an IBM summer internship and NSF Grant No. CNS-1617640. Ayten Ozge Akmandor and Niraj K. Jha are with the Department of Electrical Engineering, Princeton University, Princeton, NJ, 08544, USA, e-mail:\{akmandor, jha\}@princeton.edu. Jorge Ortiz is with the Department of Electrical and Computer Engineering, Rutgers University, Piscataway, NJ, 08854, USA, e-mail: jorge.ortiz@rutgers.edu. Irene Manotas and Bongjun Ko are with IBM Thomas J. Watson Research Center, Yorktown Heights, NY, 10598 USA, e-mail:\{Irene.Manotas, bongjun_ko\}@us.ibm.com. among labels. Thus, they are not able to exploit synergies between the feature and semantic spaces.

In this article, we show the above synergies can be exploited to improve the prediction performance of ML algorithms. Our method, called SECRET, combines vector representations of labels in the semantic space with available data in the feature space within various operations (e.g., ML hyperparameter optimization and confidence score computation) to make the final decisions (assign labels to datapoints). Since SECRET does not target any particular ML algorithm or data structure, it is widely applicable.

The main contributions of this article are as follows:

- We introduce a dual-space ML decision process called SECRET. It combines the new dimension (semantic space) with the traditional (single-space) classifiers that operate in the feature space. Thus, SECRET not only utilizes available data-label pairs, but also takes advantage of meaning-based (semantic) relationships among labels to perform classification for a given real-world task.

- We demonstrate the general applicability of SECRET on various supervised ML algorithms and a wide range of datasets for various real-world tasks.

- We demonstrate the advantages of SECRET's new dimension (semantic space) through detailed comparisons with traditional ML approaches that have the same processing and information (except semantic) resources.

- We compare the semantic space ML model with tra- 
ditional approaches. We shed light on how SECRET builds the semantic space component and its impact on overall classification performance.

The remainder of the article is organized as follows. Section 2 provides background information on Bayesian optimization and semantic vector representation of words. Section 3 provides the motivation behind SECRET's dualspace ML decision process. Section 4 introduces the methodologies underpinning the SECRET architecture, data processing, hyperparameter tuning, ML algorithm training in the feature and semantic spaces, confidence score calculation, and decision process. Section 5 presents experimental results and provides comparisons with traditional ML approaches. Section 6 presents related work from the literature and points out the novelty of SECRET. Section 7 provides a detailed discussion on SECRET from different perspectives. Finally, Section 8 discusses future research directions and concludes the article.

\section{BACKGROUND}

In this section, we discuss background material that will help with understanding of the rest of the article. We discuss Bayesian optimization for hyperparameter tuning and semantic vector representation of words.

\subsection{Bayesian Optimization for Hyperparameter Tuning}

The selected set of hyperparameter values has a direct impact on classification/regression performance. Handtuning, random search, grid search, and Bayesian optimization are commonly used methods for finding the best set of hyperparameter values. In this work, we adopt Bayesian optimization as it is known, in general, to provide an unbiased analysis and higher classification/regression performance, while requiring a small number of iterations due to the utilization of results from past iterations [4].

Bayesian optimization integrates exploration and exploitation. It starts with a prior belief over the unknown objective function. It then evaluates the optimization goal function with available data (target hyperparameter values chosen for the iteration). Based on input data and the corresponding optimization goal outputs, it updates the beliefs and selects the next set of hyperparameter values to be evaluated. The process is repeated until a maximum number of iterations is reached [5].

\subsection{Semantic Vector Models of Words}

Semantic vector models assign a compact real-valued vector to each word in a dictionary. The vector captures the word's semantic relationships with the remaining words in the dictionary. Words with close meanings are represented by closely-spaced vectors in the semantic space. Some of the algorithms that derive semantic vector word representations are Skip-gram and Continuous Bag-of-Words (CBOW) architectures of word2vec [6], GloVe [7], vLBL [8], ivLBL [8], Hellinger PCA [9], and recurrent neural networks [10].

GloVe is an unsupervised method. It uses the cooccurrence ratio of words within a pre-specified window length to obtain the word vectors. Use of this ratio enhances the distinction between two relevant words or a relevant word and an irrelevant one. The GloVe algorithm is based on weighted least squares regression. As shown in Eq. 1. it aims to minimize the difference between the scalar product of the two word vectors and the logarithm of their co-occurrence value. Weights are used to avoid dominance (overweighting) by both very frequent and rare co-occurrences. The corresponding weighting function is shown in Eq. 2. In [7], $\alpha=\frac{3}{4}$ has been found to yield good results.

$$
\begin{array}{r}
J=\sum_{i=1}^{V} \sum_{j=1}^{V} f\left(X_{i j}\right)\left(w_{i}^{T} * w_{j}+b_{i}+b_{j}-\log \left(X_{i j}\right)\right)^{2}, \\
\text { where } V: \text { vocabulary size, } \\
f(x): \text { weighting function, } \\
X: \text { co-occurrence matrix, } \\
w: \text { word vector, } \\
b: \text { bias. } \\
f(x)= \begin{cases}\left(x / x_{\max }\right)^{\alpha}, & \text { if } x<x_{\max } \\
1, & \text { otherwise }\end{cases}
\end{array}
$$

\section{Motivation}

Various features (characteristics) can be extracted from data and correlated with corresponding circumstances (labels) of interest in supervised ML. The features constitute the feature space. As an example, in healthcare, the labels may be disease names, therapy methods, or health states, whereas in the chemical industry, the labels may be chemical names, model simulation states, or stability test results. Although labels differ from one application to another, they all lead to some action being taken based on the assigned label. The action can be reporting an anomaly, continuing the process, switching states, scaling parameters, etc. Since the assigned labels impact future actions, they need to be interpretable by either humans or machines. This means that the labels also carry semantic information. However, current supervised classifiers do not take advantage of this semantic information. Consider a dataset that has 'longterm methods,' 'short-term methods,' and 'no use' as labels. As depicted in Fig. 1. current supervised ML algorithms will result in the same data-label model even if we replace the labels with 'class 1,' 'class 2,' and 'class 3.' However, 'longterm methods' and 'short-term methods' are semantically more similar but less similar to 'no use,' as indicated by the squared Euclidean distances in Fig. 2 It would be advantageous to exploit this semantic relationship during classification.

SECRET addresses the above problem through a dualspace classification approach. As shown in Fig. 3a, traditional supervised learning operates in the feature space. SECRET, on the other hand, also incorporates class affinity and dissimilarity information into the decision process, as shown by the 'Semantic space' block in Fig. 3b This property enables SECRET to make informed decisions on class labels. SECRET makes the final decision (labeling) by integrating information from both the feature and semantic spaces. Therefore, it is able to deliver higher classification performance relative to traditional approaches because of 


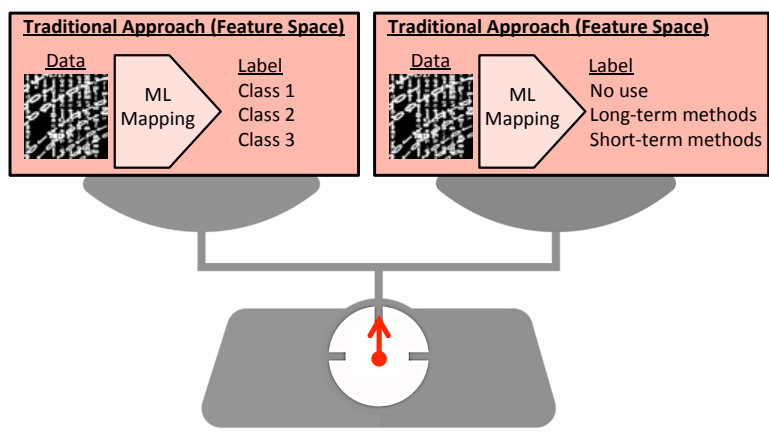

(a)

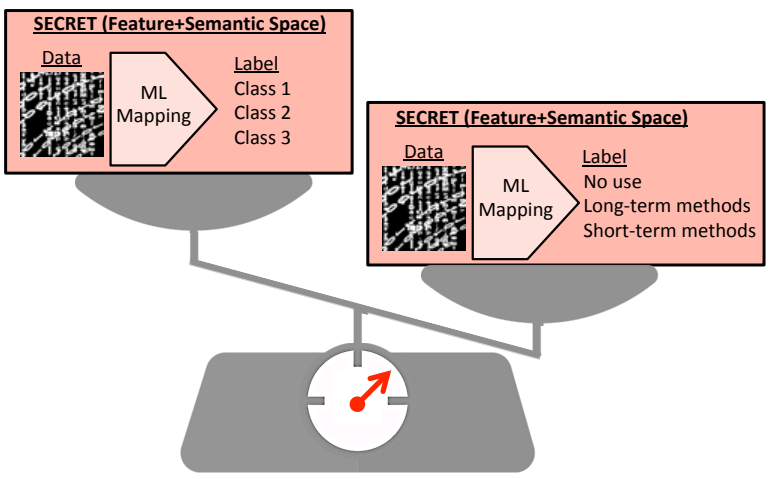

(b)

Fig. 1. ML data-label mapping responses of (a) the traditional approach and (b) SECRET. The scale signifies whether the data-label models are equal (equilibrium state) for the two types of labels.

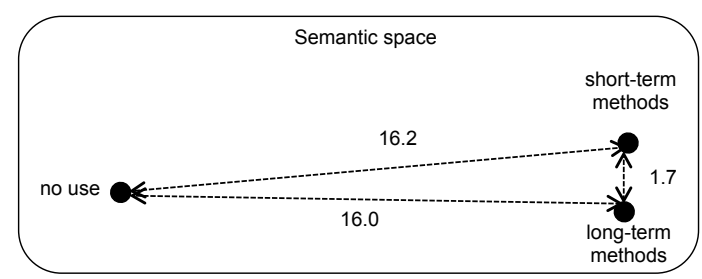

Fig. 2. Pairwise squared Euclidean distances in between the vector representations of labels in the semantic space. A smaller distance indicates a larger class affinity.

its reliance on a richer semantic+feature space. Although this example only exploits the semantic+feature space, there may be other as-yet-undiscovered spaces that could also be integrated into SECRET in a similar fashion.

\section{Methodology}

In this section, we describe SECRET's data processing and dual-space classification procedure in detail.

\subsection{The SECRET Architecture}

SECRET integrates information from two sources: feature space and semantic space.

- The feature space includes data, extracted features (if available), and the corresponding labels.

- The semantic space includes meaning-based relationships among labels in the form of real-valued word vectors.

As shown in Fig. 4a traditional supervised learning operates in the feature space. It uses the features to model the data-label relationship. On the other hand, SECRET not only

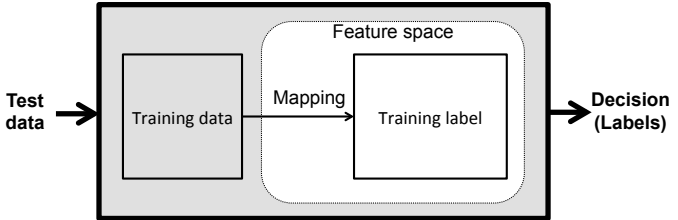

(a)

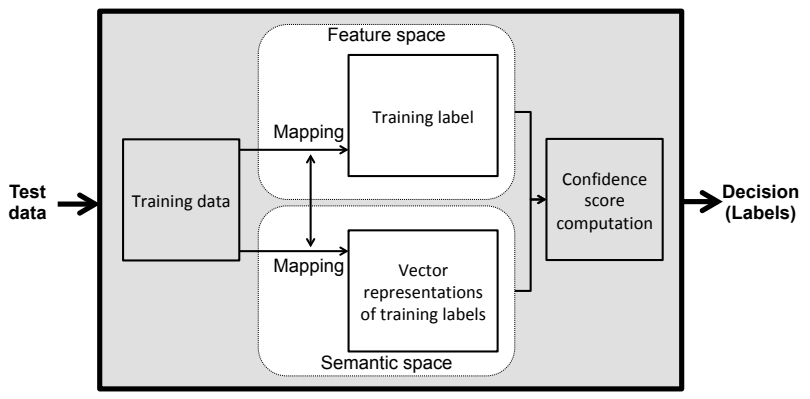

(b)

Fig. 3. Classification methods: (a) traditional and (b) SECRET.

uses data available in the feature space, but also integrates meaning-based relationships among labels (semantic space) into the decision process, as shown in Fig. $4 b$ SECRET requires vector representations of the training labels as an additional input, relative to the traditional supervised learning approach. Vector representations are obtained using semantic vector generation algorithms (see Section 2.2) that are trained with a large number of documents. Depending on the available computational resources, SECRET can be implemented with either pre-trained semantic vectors that are available on the web [11], [12], [13], or specially-trained semantic vectors obtained from a given corpus. Neither implementation needs the involvement of an expert, unlike the case of labeling data in supervised learning.

The novelty of SECRET is that it enables an interaction between the two spaces while constructing the classifiers and regressors. In Fig. $4 b$ the interaction is depicted by the arrow in between the two spaces. The hyperparameter values of the semantic (feature) space are not aimed at maximizing the performance of the semantic space regressor (classifier), but that of the overall SECRET architecture. However, the interaction does not only take place during hyperparameter tuning. Unlike the traditional approaches, the classifier and regressor do not make individual decisions. Both provide confidence scores for each label. This information is used by SECRET to predict the label for a new query data instance. We explain each block next.

\subsection{Data Processing}

Data processing is an important part of any ML decision process. Data in the raw form require: (i) denoising [14], [15], [16], [17], (ii) outlier elimination [18], (iii) feature extraction [19], (iv) feature encoding [19], and (v) normalization/standardization [19]. SECRET targets these operations in the 'Data Processing' block in Fig. 4b. For more details, readers are referred to the cited references.

\subsection{Hyperparameter Tuning}

The selected set of hyperparameter values has a direct impact on classification/regression performance. In this work, 


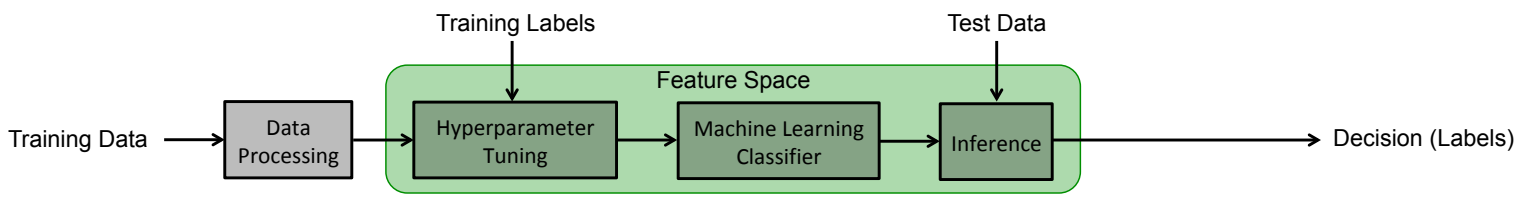

(a)

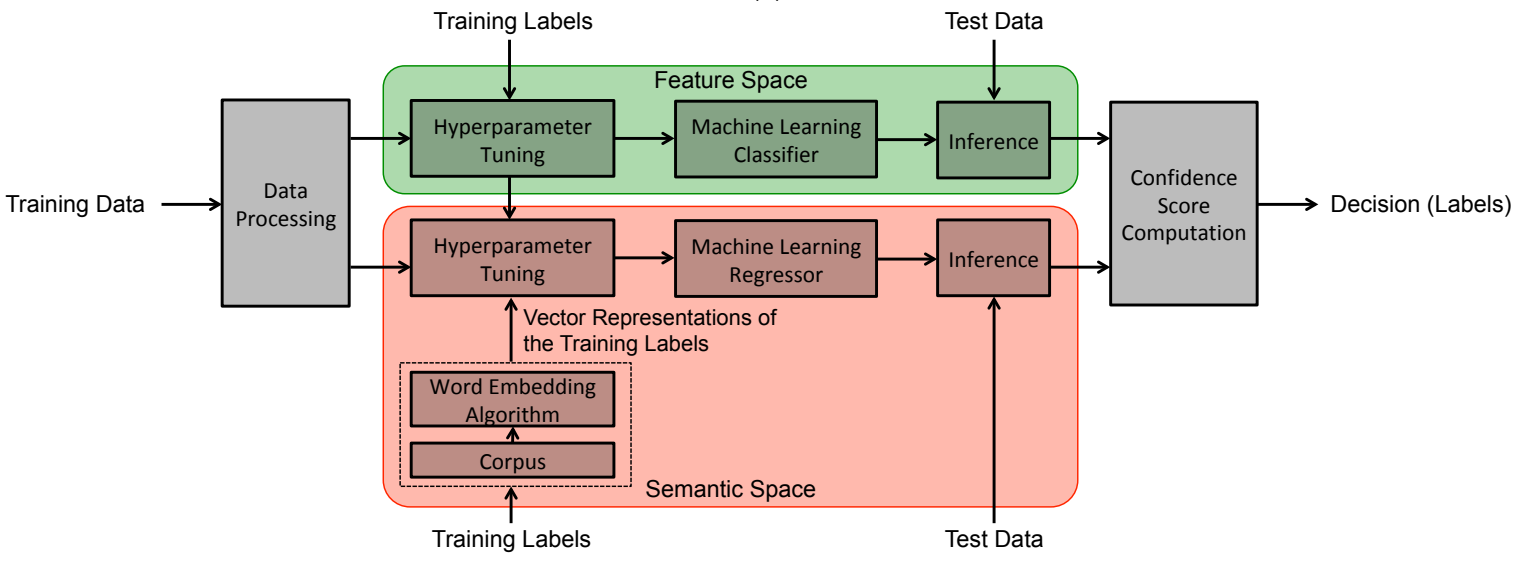

(b)

Fig. 4. Architectures: (a) traditional supervised learning and (b) SECRET.

we adopt Bayesian optimization for reasons mentioned in Section 2.1.

By integrating exploration and exploitation, Bayesian optimization outputs the set of hyperparameter values that maximizes the optimization goal function. This function indicates the overall performance of the chosen supervised ML algorithm. Therefore, it guides Bayesian optimization to find the appropriate set of hyperparameter values in order to enhance the performance of real-world decision processes. The pseudocode for the hyperparameter tuning stage is shown in Algorithm 1 . Following preprocessing of training and validation data, the Gaussian Process (GP) of Bayesian optimization is initialized. Bayesian optimization takes hyperparameters (as variables, not their values), their ranges, and optimization goal function as input. The hyperparameters depend on the chosen ML algorithm. For example, whereas the total number of trees may be a hyperparameter for the random forest (RF) algorithm, the number of layers and neurons in each layer may be hyperparameters for the multi-layer perceptron (MLP) algorithm. The optimization goal function reflects the purpose of the task being performed. Depending on whether SECRET is implemented on top of a traditional supervised (feature space) classifier or built from the ground up, the optimization goal function takes into account either available feature and semantic space hyperparameter values or both semantic and feature space hyperparameter values. The function outputs performance metrics, such as accuracy, F1 score, etc., based on training and validation data. We implement SECRET on top of a traditional supervised classifier in order to compare it with classification algorithms from the literature. Therefore, in Algorithm 1, the feature and semantic space algorithms are trained with already assigned hyperparameter values and acquisition function outputs (semantic space hyperparameter values), respectively. Then the feature and semantic space confidence scores are calculated and labels are assigned (see Section 4.5 for details). The optimization goal function output (classification performance on validation set) is used to update the beliefs and obtain the next set of semantic space hyperparameter values. The above process is repeated with this new set. When the maximum number of allowed iterations (BOiter) is reached, the process is stopped and the set of hyperparameter values (SSHyp) that leads to the highest validation set performance is selected for application to the test set.

\subsection{Training of ML Models and Inference}

The data-label relationships exist in different forms in the feature and semantic spaces and are captured through ML algorithms. The feature space does not take into account the meaning-based relationships among labels. However, the semantic space takes into account the affinity and dissimilarity information between labels that is captured in a vector form. Hence, whereas the feature space decision process maps data to the label with the help of a classifier, the semantic space decision process relies on a regressor. The choice of the regressor has a direct impact on SECRET's performance. Thus, for a fixed feature space classifier, we carry out performance analyses with various regressors on the training and validation data and select the one that best maps data to labels. After finding the best set of hyperparameter values in both spaces through joint optimization, we train the ML algorithms. We train the feature space classifier with the selected hyperparameter values, training data, and training labels. Then we train the semantic space regressor with the selected hyperparameter values, training data, and vector representations of the labels. Following the training stage, we perform inference on the test data and obtain the confidence scores. We show the operations corresponding to this stage in lines 3 through 6 in Algorithm 2

\subsection{Confidence Score Computation and Decision}

The inference stage outputs the confidence scores for each data instance for both spaces. The feature space confidence 
score (FSConf) computation depends on the chosen ML algorithm. For example, the confidence score of the RF classifier is computed as the average class probabilities of all trees. The class probability for each tree is computed as the fraction of the samples that belong to the class of interest in a leaf. However, the confidence score of the MLP classifer is the same as the output of the activation function in the outermost (final) layer. On the other hand, as shown in Fig. 5 the semantic space confidence score, SSConf, is based on Euclidean distance, in line with the main motivation behind semantic vector representations. The distance between the vector representation of the class label and the assigned vector is inversely proportional to $S S C o n f$. As shown in Eq.3. $S S C o n f$ is computed through the normalized inverse ratio of the squared distance between the assigned vector and label vector. In Algorithm 2, the corresponding operations are shown in lines 7 through 9. $D, V$, and $C$ represent the dimension of the semantic word vector, semantic vector of the class label, and total number of classes, respectively. $\epsilon$ refers to additive shift. In line $9, \epsilon$ is used to avoid divergence of the algorithm when the assigned vector (regressor output) overlaps with the vector of the class label. The value of $\epsilon$ needs to be smaller than the minimum difference between vectors of the assigned label and the class label. However, in the hyperparameter tuning stage (line 14 in Algorithm $1, \epsilon$ is assigned 0 and divergence is permitted to avoid overfitting. Since divergence blocks label assignment, an overlap between the assigned and class labels degrades classification performance on the validation set significantly. Therefore, the hyperparameters corresponding to this case are not selected (line 17 through line 19 in Algorithm 1) and overfitting is avoided. In summary, while assigning 0 to $\epsilon$ in the hyperparameter tuning stage is beneficial for preventing overfitting, a nonzero value in the decision making process is needed to avoid divergence.

$$
\begin{array}{r}
S \operatorname{SConf} f_{k}=\frac{\frac{1}{d_{k}^{2}+\epsilon}}{\sum_{i=1}^{C} \frac{1}{d_{i}^{2}+\epsilon}}, k \in[1, C], \\
\text { where C: Total number of classes, }
\end{array}
$$

$d$ : Distance between assigned vector and class label,

$$
\epsilon \text { : Additive shift. }
$$

The overall confidence score is computed by taking the average of FSConf and SSConf for each class (Fig. 6). In the decision stage, each data instance in the test set is assigned the label of the class that has the highest overall confidence score (line 10 in Algorithm 22. Following the labeling stage, SECRET's classification performance is assessed through accuracy $(A C C)$ and F1 score metrics computed using Eq. 4 and Eq. 5. respectively. Accuracy depicts the ratio of the number of correctly classified instances and the total number of instances. However, the F1 score indicates the fraction of correctly classified instances for each class within the dataset.

$$
A C C=\frac{T P+T N}{T P+T N+F P+F N}
$$

where $T P$ : True positive, $T N$ : True negative,
Algorithm 1 SECRET - Hyperparameter Tuning

Input:

DataTr: Training data

DataVal: Validation data

LabelTr: Labels corresponding to training data

LabelVal: Labels corresponding to validation data

FSHyp: Feature space hyperparameter values

$V$ : Vector representations of the labels

\section{Output:}

SSHyp: Semantic space hyperparameter values

1: Preprocess DataTr, See Section 4.2

2: Preprocess DataVal, See Section 4.2

3: $A C C_{\max } \leftarrow 0$

4: SSHyp $\leftarrow$ null

5: Initialize GP of Bayesian Optimization

6: for $i=1, \ldots$, BOiter

7: $\quad$ FSModel $\leftarrow$ FSClassifier(DataTr,LabelTr,FSHyp)

8: $\quad F S C o n f \leftarrow F S M o d e l($ DataVal $)$

9: $\quad$ SSHyp $\leftarrow$ Exploration+Exploitation

10: $\quad$ SSModel $_{i} \leftarrow$ SSRegressor (DataTr, LabelTr,SSHyp $\left.i\right)$

11: $\quad$ SSOut $\leftarrow$ SSModel $_{i}$ (DataVal $)$

12: $\quad$ for $j=1, \ldots$, \#instances

13: $\quad$ for $k=1, \ldots, C$

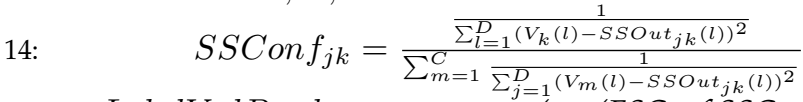

15: $\quad$ LabelValPred $\leftarrow \operatorname{argmax}_{\text {class }}(\operatorname{avg}($ FSConf,SSConf $))$

16: $\quad A C C_{i} \leftarrow A C C($ LabelVal,LabelValPred $)$

17: $\quad$ if $A C C_{i}>A C C_{\max }$

18: $\quad A C C_{\max }=A C C_{i}$

19: $\quad S S H y p=S S H y p_{i}$

20: $\quad$ Update $G P$

21: return $S S H y p$

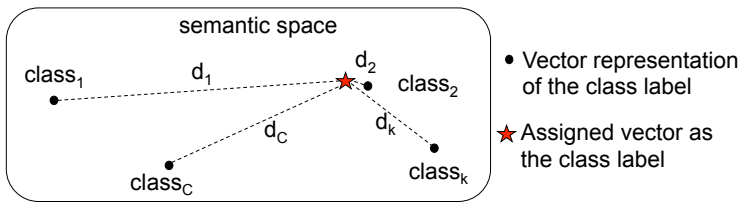

Fig. 5. Confidence score computation in the semantic space. Confidence score is calculated for each $k$, where $k \in[1, C]$ and $C$ represents the total number of classes.

$$
\begin{array}{r}
F 1=\frac{\sum_{i=1}^{C} 2 * P R E C_{i} * R E C_{i} /\left(P R E C_{i}+R E C_{i}\right)}{C}, \\
\text { where } P R E C=\frac{T P}{T P+F P}, R E C=\frac{T P}{T P+F N}, \\
C: \text { Total number of classes, } \\
P R E C: \text { Precision, } R E C: \text { Recall. }
\end{array}
$$

\section{Experimental Results and Discussion}

In this section, we present the experimental results for SECRET and provide comparisons with traditional supervised classifiers and ensemble methods. Then, we analyze the effect of feature-semantic space variations on SECRET's classification performance. 
Algorithm 2 SECRET - ML Training - Inference - Decision Input:

DataTrVal: Training and validation data

DataTe: Test data

LabelTrVal: Labels corresponding to training and validation data

LabelTe: Labels corresponding to test data

FSHyp: Feature space hyperparameter values

SSHyp: Semantic space hyperparameter values

$V$ : Vector representations of the labels

Output:

ACC: SECRET's accuracy on the test set

F1: SECRET's F1 score on the test set
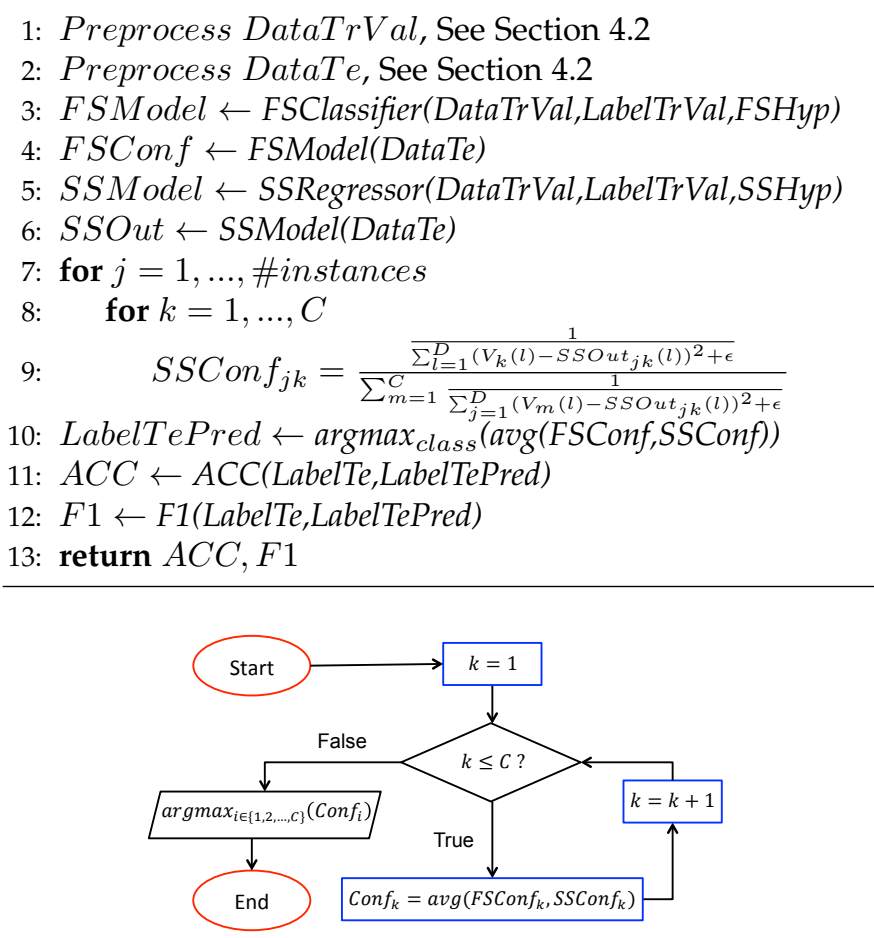

Fig. 6. Overall confidence score computation and decision-making stages of SECRET.

\subsection{Datasets}

SECRET's flexible design ensures applicability to a broad spectrum of real-world classification tasks. We analyze its performance on datasets for ten different applications, ranging from biomedical disease diagnosis to sonar-based object detection. Table 1 describes these datasets and their characteristics. The datasets are taken from the UCI Machine Learning Repository [20]. They focus on the classification task.

The UCI Connectionist Bench Dataset is based on sonar signals that are reflected from a rock or metal cylinder. It discriminates between these two obstacles. The UCI Indian Liver Patient Dataset is composed of patient records, such as age, gender, total bilirubin, total protein, albumin, etc. It is aimed at diagnosing liver disease. The UCI Breast Cancer Wisconsin Dataset is built using images of a fine needle aspiration of the breast. It focuses on classifying the cell nucleus as malignant or benign. The Statlog Dataset is composed of physiologic signal and demographic information of patients. It is aimed at predicting the absence or presence of heart disease. The UCI Contraceptive Method Choice Dataset is composed of demographic and socio-economic information of married women. It is aimed at identifying their contraceptive method choices. The UCI Lymphography Dataset includes features extracted from lymphography images. It is aimed at classifying different types of lymph nodes. The UCI Nursery Dataset includes parental occupation, child's nursery condition, family structure, and the family's social, health, and financial status as features. It is targeted at ranking of nursery school applications. The UCI Cardiotocography Dataset is formed using cardiotocography features that are based on fetal heart rate and uterine contraction. It focuses on classification of ten different fetal morphologic patterns. The UCI Chess Dataset is built using the king-rook and rook positions on a chessboard. It is targeted at the depth of a win. The UCI Letter Recognition Dataset is formed using black-and-white image pixels of letters of the English alphabet. It is targeted at classifying each of the 26 letters.

\subsection{Supervised Classifier vs. SECRET}

We hypothesize that feature space information is not the only source of information that can be used for classification. In order to test this hypothesis, we compare the classification performance of the supervised classifier with that of SECRET. The supervised classifier uses feature space information to model the data-label relationship and predict labels of unlabeled data instances. On the other hand, SECRET fuses the feature and semantic space information to predict labels. By analyzing the two approaches, we aim to identify the impact of semantic space information on classification performance. In order to minimize dependency on an ML algorithm, we use two classifiers (RF and MLP) and their regressor versions. Decision trees in RF are information-based; however, MLP is error-based. Second, in order to avoid a biased evaluation of classification performance, we compute both accuracy and F1 scores by comparing the predicted labels with actual ones in the test set. Accuracy reflects the percentage of correctly classified samples within the test set. However, the F1 score incorporates precision and recall values, which are computed from the false positive, false negative, and true positive values for each class, and then taking the average. Therefore, accuracy and F1 score assess the classification performance from different perspectives.

We implement SECRET with a Bayesian optimization framework [21] (used for determining the number of neurons in an MLP with a single hidden layer and the number of trees in RF), scikit-learn [22], and 50-dimensional GloVe vectors |11| (pretrained with Wikipedia 2014 + Gigaword 5). In the case of a label with more than one word, we take the average of the word vectors corresponding to the words within the label to find the overall label vector. We compute the semantic space confidence score using the equations shown in line 14 of Algorithm 1 and line 9 of Algorithm 2. Based on our analyses (see the conditions mentioned in Section 4.5, $\epsilon$ is assigned $10^{-200}$ in the experiments. For classification performance analyses, we use stratified 10-fold sampling for each dataset and report the average accuracy and F1 score values.

Fig. 7 shows the legend for the plots that depict classification performance of the traditional feature space approach 
TABLE 1

Datasets and Their Characteristics

\begin{tabular}{|c|c|c|c|c|c|}
\hline Dataset & Abbreviation & \# Instances & \# Features & \# Classes & Class Labels \\
\hline $\begin{array}{l}\text { UCI Connectionist Bench (Sonar, Mines } \\
\text { vs. Rocks) Dataset }\end{array}$ & sonar & 208 & 60 & 2 & Rock, Metal cylinder \\
\hline UCI ILPD (Indian Liver Patient Dataset) & liver & 583 & 10 & 2 & Liver patient, Not liver patient \\
\hline UCI Statlog (Heart) Dataset & heart & 270 & 13 & 2 & Absence, Presence \\
\hline $\begin{array}{l}\text { UCI Contraceptive Method Choice } \\
\text { Dataset }\end{array}$ & $\mathrm{cmc}$ & 1473 & 9 & 3 & $\begin{array}{l}\text { No use, Short-term methods, Long-term } \\
\text { methods }\end{array}$ \\
\hline UCI Nursery Dataset & nursery & 12960 & 8 & 5 & $\begin{array}{l}\text { Not recommended, Recommended, Very } \\
\text { recommended, Priority, Special Priority }\end{array}$ \\
\hline UCI Cardiotocography Dataset & cardio & 2126 & 21 & 10 & $\begin{array}{l}\text { Calm sleep, REM sleep, Calm vigilance, } \\
\text { Active vigilance, Shift pattern, Stress sit- } \\
\text { uation, Vagal stimulation, Largely vagal } \\
\text { stimulation, Pathological state, Suspect } \\
\text { pattern }\end{array}$ \\
\hline UCI Letter Recognition Dataset & letter & 20000 & 16 & 26 & $\begin{array}{l}\text { A, B, C, D, E, F, G, H, I, J, K, L, M, N, O, } \\
\text { P, Q, R, S, T, U, V, W, X, Y, Z }\end{array}$ \\
\hline
\end{tabular}

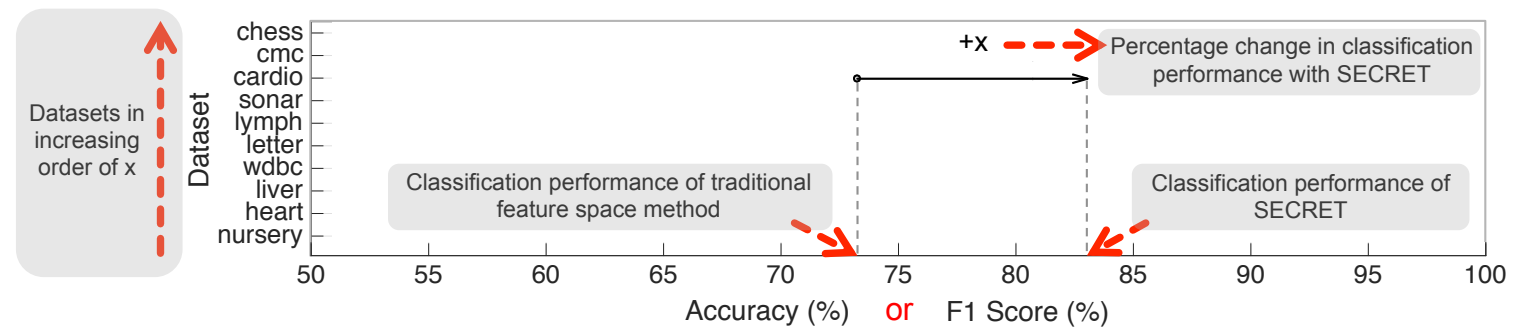

Fig. 7. Legend for experiments that compare traditional approches with SECRET.

and SECRET. The starting points of the arrows indicate accuracy or F1 scores of the feature-space classifiers. The ending points indicate the impact of including semantic space information on classification. The numbers above/below the arrows show the percentage improvement. The arrow sizes are scaled accordingly. Dataset names are ordered based on the amount of change in classification performance caused by SECRET. The dataset on top shows the largest change. The amount of change decreases from top to bottom.

Fig. 8 shows the accuracy and F1 scores of the traditional MLP classifier and SECRET with the format shown in Fig. 7 In this case, SECRET integrates semantic information into the MLP classifier with the help of either an RF or MLP regressor. It chooses the type of regressor based on validation set performance, builds the overall classifier using both the semantic and feature spaces, and makes the final decision on the test labels. The color of arrows in Fig. 8 indicates the chosen regressor type. If both black and dark blue colored arrows are shown for a dataset, then the validation set classification performance is inconclusive (both regressors perform equally well on the validation set with less than or equal to $1.0 \%$ difference in accuracy and F1 score values) in determining the better regressor. We present results with both regressors. The chess dataset has $14.0 \%$ and $13.1 \%$ improvements in accuracy and F1 scores, respectively. While the liver dataset has $1.2 \%$ improvement in accuracy, it has the second highest F1 score improvement of $13.0 \%$ among all datasets. This shows the importance of analyzing classification performance from different perspectives. When there is class imbalance, F1-macro score shines light on classification performance of the minority class. As in the case of the liver dataset, biomedical disease diagnosis datasets are likely to be imbalanced. Although the main goal is to detect the disease, in general, the 'healthy' class has more datapoints than the disease one. Class imbalance might affect performance. However, it does not only affect the performance of the feature space classifier, but also that of SECRET. We upsampled the minority class with the SMOTE method and repeated the experiments to test this point. While the feature space resulted in $60.3 \%$ accuracy and $58.1 \%$ F1 score, SECRET led to $69.8 \%$ accuracy and $65.0 \%$ F1 score. Although upsampling improved classification performance of both approaches, SECRET's accuracy and F1 score dominated the traditional feature space classifier by $9.5 \%$ and $6.9 \%$, respectively. Moreover, except for the lymph and heart datasets, we observe that the arrows point to the right, indicating that SECRET improves accuracy as well as the F1 score. More specifically, use of the RF or MLP regressor, choice depending on the validation set performance, in the semantic space leads to classification performance improvement in nine out of ten and eight out of ten datasets, respectively. The amount of improvement 


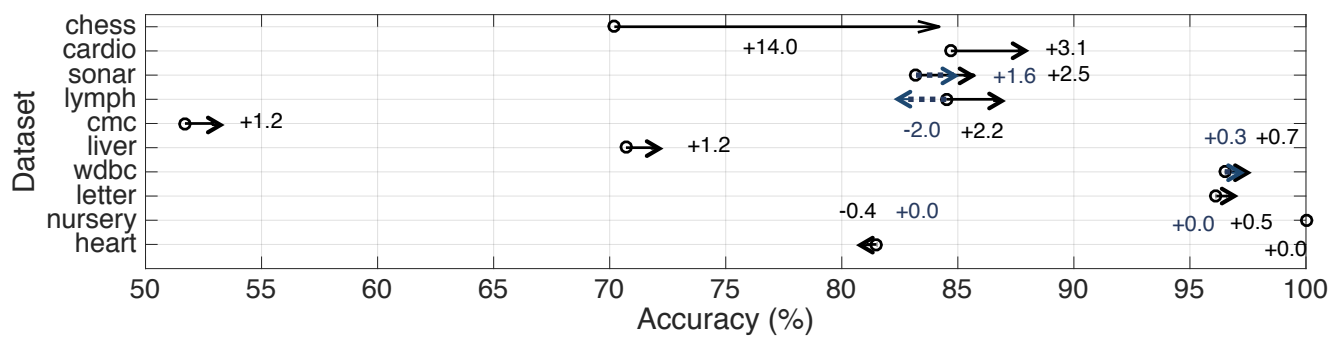

(a)

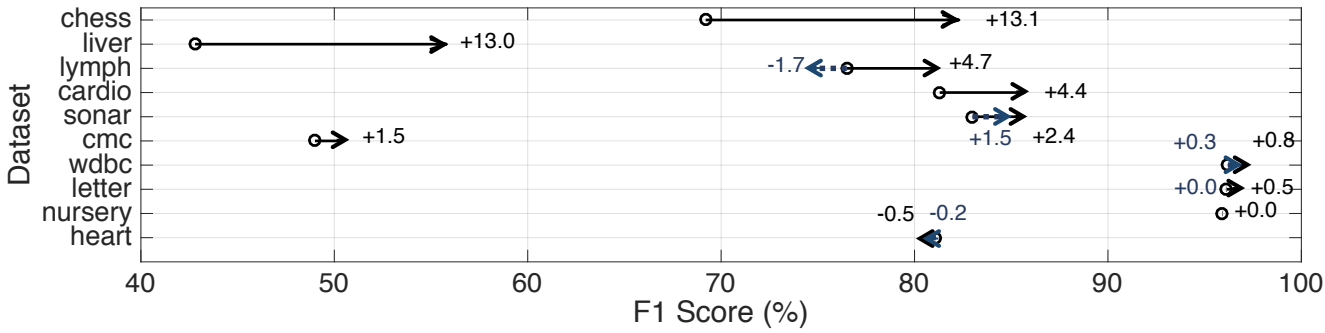

(b)

Fig. 8. SECRET's (a) accuracy and (b) F1 score improvements over the traditional MLP (feature-space) classifier. SECRET uses MLP as the feature space classifier and RF/MLP as the semantic space regressor. Black arrows indicate when the RF regressor is used, whereas the dark blue and dashed arrows correspond to the MLP regressor.

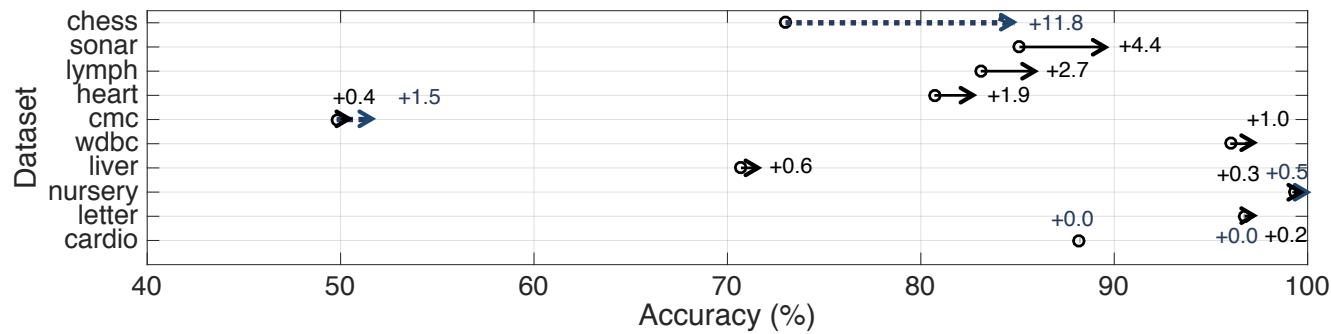

(a)

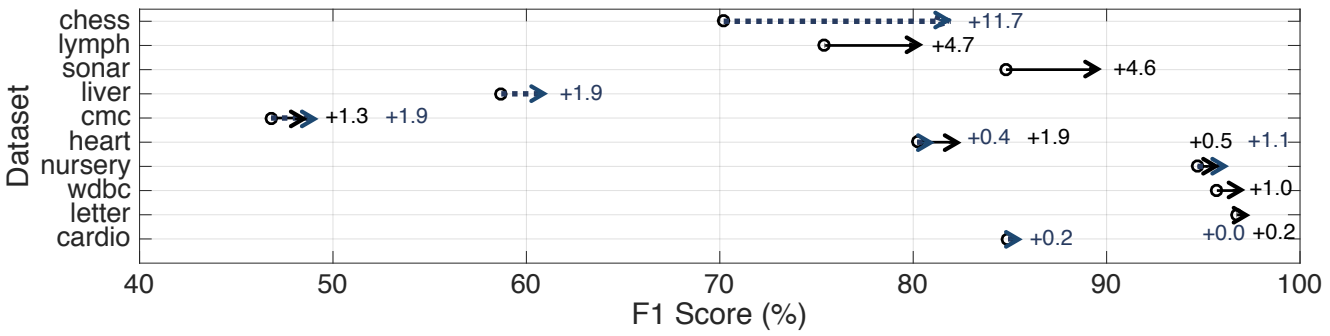

(b)

Fig. 9. SECRET's (a) accuracy and (b) F1 score improvements over the traditional RF (feature-space) classifier. SECRET uses RF as the feature space classifier and MLP/RF as the semantic space regressor. Black arrows indicate when the MLP regressor is used, whereas the dark blue and dashed arrows correspond to the RF regressor.

depends on dataset characteristics, feature space classifier, and chosen semantic space regressor.

Fig. 9 shows the classification performance of the traditional RF classifier and SECRET implemented with an MLP or RF regressor. In this experimental setup, SECRET shows $11.8 \%$ accuracy and $11.7 \%$ F1 score improvements for the chess dataset. Moreover, SECRET achieves $4.4 \%$ accuracy and $4.6 \%$ F1 score improvements for the sonar dataset. For the lymph dataset, we observe a $2.7 \%$ accuracy and $4.7 \%$ F1 score improvement with the MLP regressor. For the liver dataset, as in the case of Fig. 8, a $1.9 \%$ increase in the F1 score points to the positive impact of the semantic space information on decreasing the false positive and false negative rates, thus increasing the precision and recall values. None of the arrows in Fig. 9 point to the left, confirming the stable performance enhancement of SECRET that is independent of application type or dataset.

Overall, the results shown in Fig. 8 and Fig. 9 demonstrate classification performance improvement with SECRET.

\subsection{Ensemble Method vs. SECRET}

We saw in the previous section that SECRET outperforms traditional supervised ML classifiers. However, the traditional classifier also can be made more robust by using an ensemble method. In this section, we compare ensemble methods with SECRET to show that the semantic space offers a different type of information source that pays rich dividends. In order to have a fair comparison between traditional ensemble methods and SECRET, we 


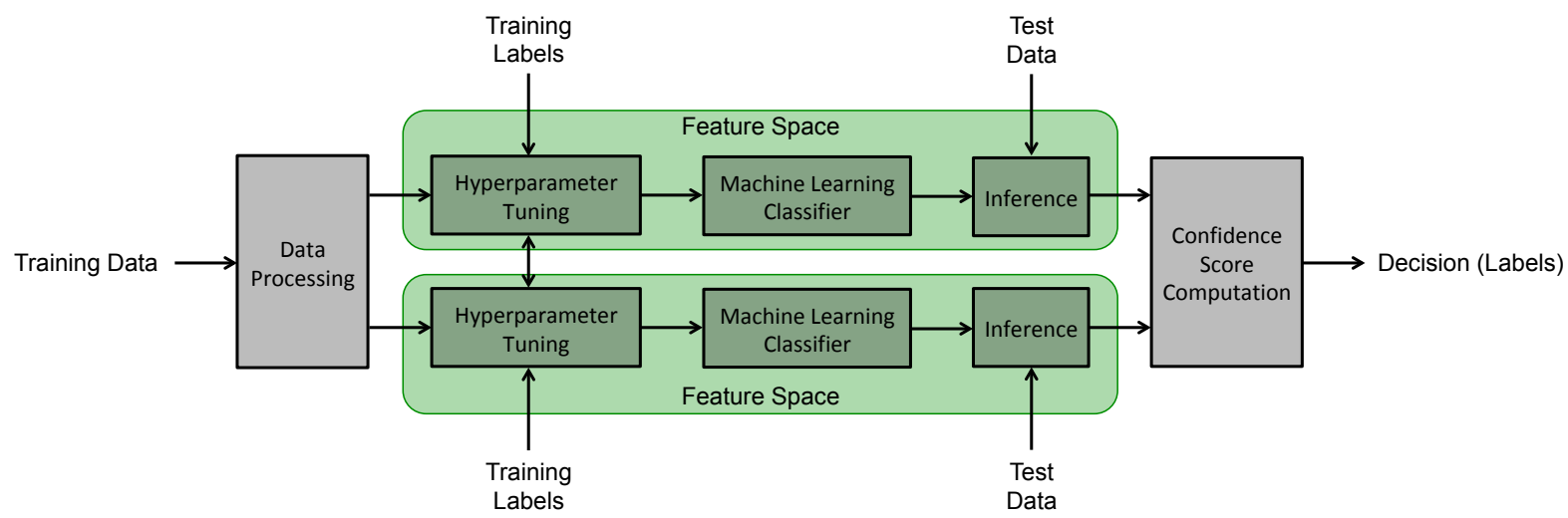

Fig. 10. Architecture of the traditional ensemble method.

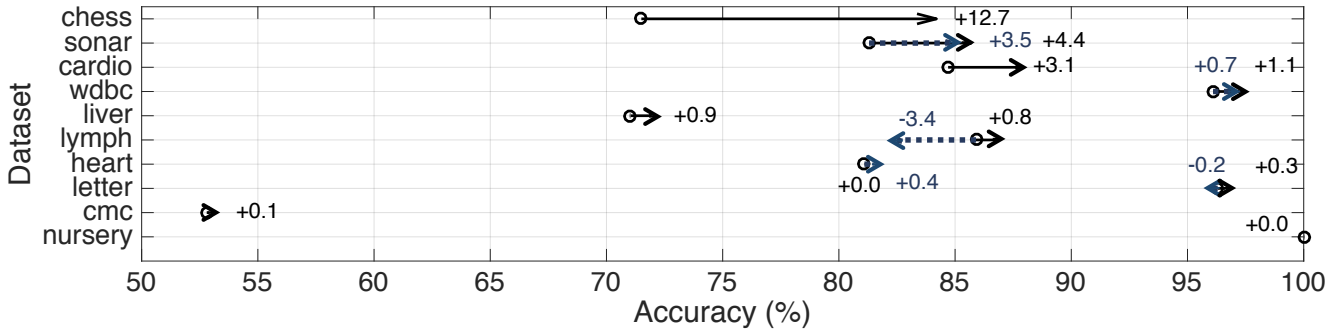

(a)

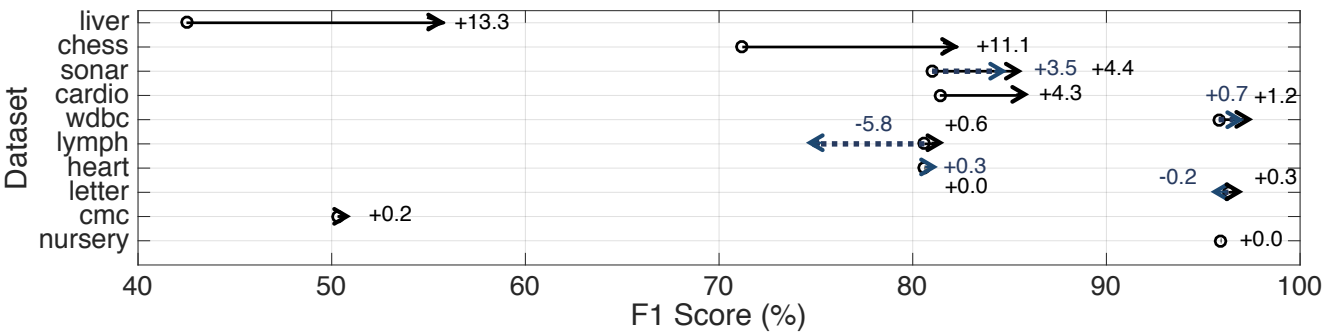

(b)

Fig. 11. SECRET's (a) accuracy and (b) F1 score improvements over the traditional MLP-MLP ensemble in the feature space. SECRET uses MLP as the feature space classifier and RF/MLP as the semantic space regressor. Black arrows indicate when the RF regressor is used, whereas the dark blue and dashed arrows correspond to the MLP regressor.

replace the red 'Semantic Space' block in Fig. $4 \mathrm{~b}$ with a 'Feature Space' block. The corresponding block diagram for the ensemble method is shown in Fig. 10 The ensemble method is composed of only feature space classifiers. In the experiments, we provide the same amount of processing, hyperparameter tuning, and decision-making resources to the two approaches. The only difference is that only the feature space information is used in the ensemble method, whereas both the feature and semantic space information is used in SECRET. We analyze the ensembles (formed with MLP and RF algorithms) and compare them with SECRET next.

Fig. 11 shows the accuracy and F1 scores of the traditional ensemble method and SECRET on the ten datasets. The ensemble is built using an MLP classifier whose performance is maximized with the best set of hyperparameter values. Then this classification performance is enhanced by combining the classifier with another MLP with hyperparameter values that maximize the overall performance of the ensemble. SECRET is built in the same way. However, the feature space classifier is replaced with a regressor that models the data and semantic vector relationship. Since the lymph dataset has the smallest size, the use of one regressor or the other leads to a significant classification performance degradation or some improvement. Due to this instability, we do not use the lymph dataset to come to a conclusion. For seven datasets (chess, sonar, cardio, wdbc, liver, heart, and cmc), SECRET achieves a 0.1 to $12.7 \%$ higher accuracy and a 0.2 to $13.3 \%$ higher F1 score relative to the ensemble method. For the nursery dataset, both approaches show comparable classification performance. Also, as in the experiments described in Section 5.2. while the liver dataset has a $0.9 \%$ increase in accuracy with SECRET, it obtains the highest F1 score improvement of $13.3 \%$. Overall, as indicated by rightward-pointing arrows, SECRET can be seen to outperform the ensemble method.

Fig. 12 shows individual and relative classification performance of the MLP-RF ensemble and SECRET. Again, the lymph dataset shows performance instability due to its size. For chess, cmc, liver, sonar, and cardio datasets, SECRET improves classification performance, whereas for the rest (excluding lymph), SECRET either obtains the same or less than $0.8 \%$ lower performance relative to the ensemble method. While SECRET improves the accuracy by 0.2 to $7.6 \%$ for the five datasets (excluding lymph), the ensemble method only outperforms SECRET by a maximum of $0.8 \%$ in the case of two datasets. SECRET and the ensemble method obtain comparable performance for the nursery and 


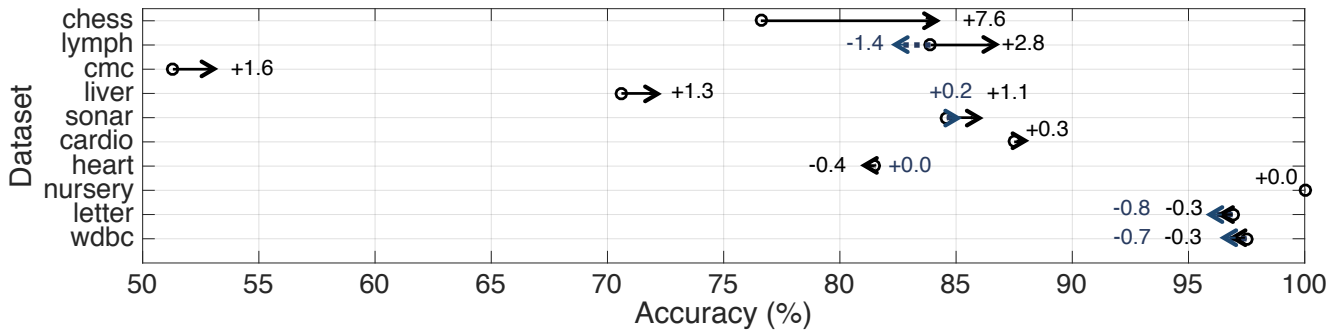

(a)

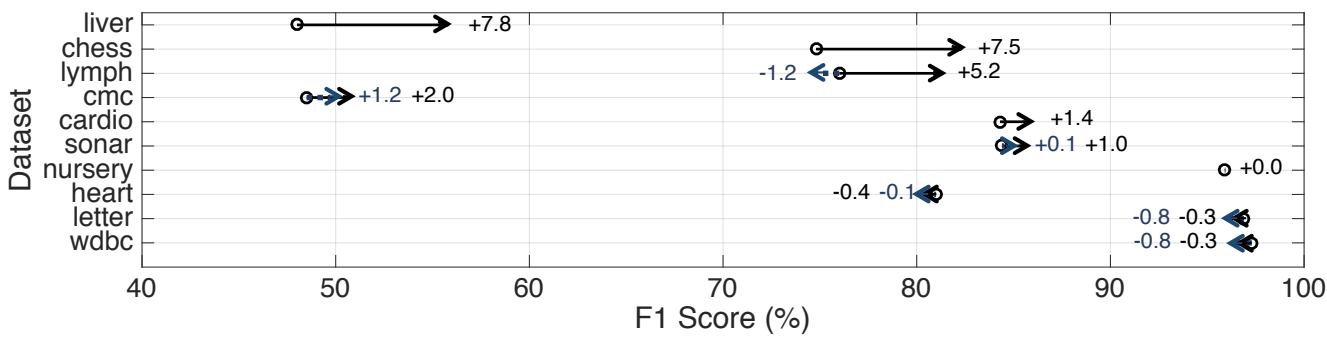

(b)

Fig. 12. SECRET's (a) accuracy and (b) F1 score improvements over the traditional MLP-RF ensemble in the feature space. SECRET uses MLP as the feature space classifier and RF/MLP as the semantic space regressor. Black arrows indicate when the RF regressor is used, wheras the dark blue and dashed arrows correspond to the MLP regressor.

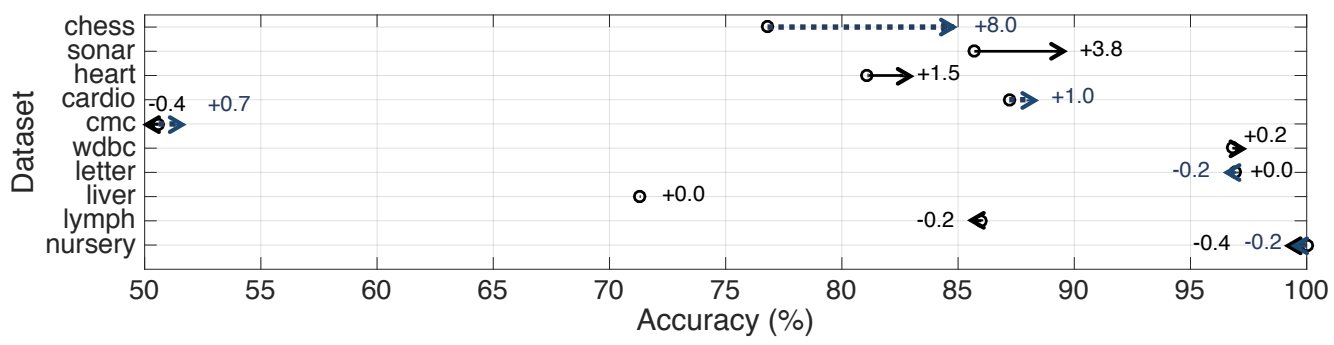

(a)

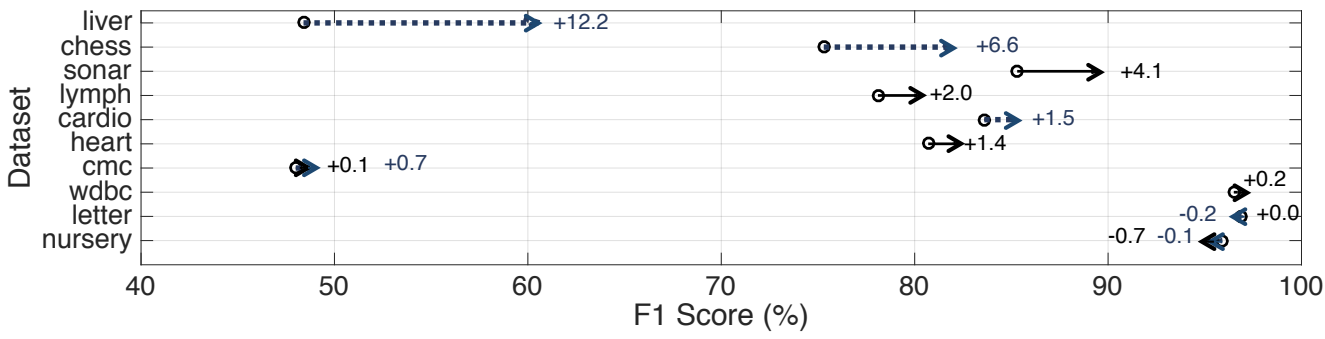

(b)

Fig. 13. SECRET's (a) accuracy and (b) F1 score improvements over the traditional RF-MLP ensemble in the feature space. SECRET uses MLP as the feature space classifier and MLP/RF as the semantic space regressor. Black arrows indicate when the MLP regressor is used, whereas the dark blue and dashed arrows correspond to the RF regressor.

heart datasets.

Fig. 13 presents accuracy and F1 scores of the RF-MLP ensemble and SECRET. If we had not implemented SECRET on top of a traditional supervised (feature space) classifier, but built it from ground up, the MLP-RF ensemble would yield the same results as RF-MLP. However, since we would like to compare SECRET with the traditional approach, the hyperparameter values are determined by also taking into account the assigned hyperparameter values of the feature space block. Since SECRET determines the semantic space hyperparameters using joint information from the two spaces, for a fair comparison, we provide the same opportunity to the ensemble method while determining the hyperparameter values of the second feature space block. Therefore, while RF hyperparameter values take advan- tage of the knowledge of MLP hyperparameter values in Fig. 12. MLP hyperparameter values take advantage of the knowledge of RF hyperparamenter values in Fig. 13 Due to very similar validation set performance ( $\leq 1 \%$ difference), we were not able to determine the regressor type for the nursery and cmc datasets. Therefore, we present both results for SECRET with RF and MLP regressors. Although the accuracy improvement of SECRET on the $\mathrm{cmc}$ dataset is inconclusive, SECRET obtains consistent F1 score improvements with both regressors. The lymph dataset shows very slight decrease $(0.2 \%)$ in accuracy; however, improvement $(2.0 \%)$ in F1 score with SECRET. For the letter and nursery datasets, the ensemble method has less than $0.1 \%$ to $0.7 \%$ higher F1 score, whereas SECRET has a 0.1 to $12.2 \%$ F1 score improvement on the remaining eight datasets. 


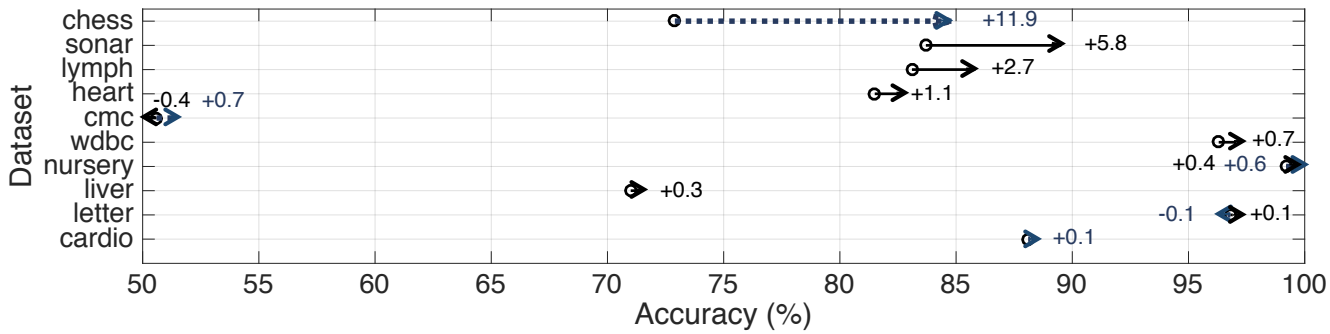

(a)

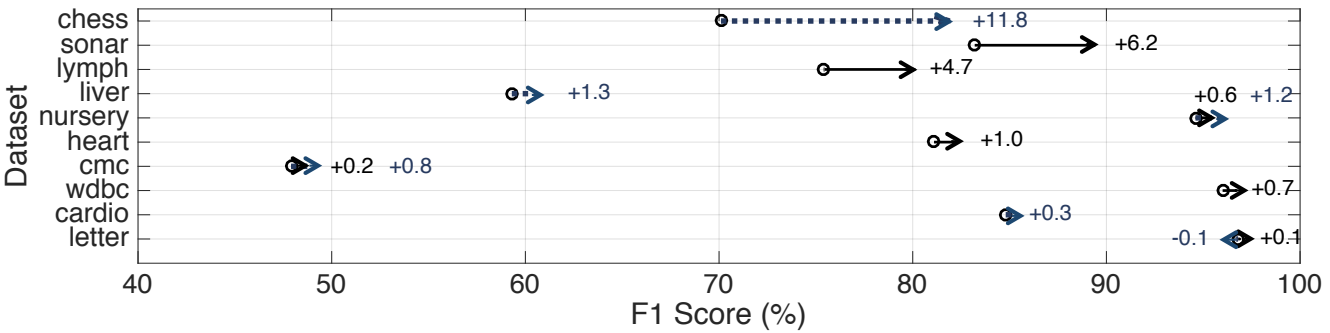

(b)

Fig. 14. SECRET's (a) accuracy and (b) F1 score improvements over the traditional RF-RF ensemble in the feature space. SECRET uses MLP as the feature space classifier and MLP/RF as the semantic space regressor. Black arrows indicate when the MLP regressor is used, whereas the dark blue and dashed arrows correspond to the RF regressor.

Fig. 14 shows the experimental results for the RF-RF ensemble and SECRET. For the letter dataset, SECRET obtains comparable performance with the ensemble method. For the remaining nine datasets, SECRET provides up to $11.9 \%$ and $11.8 \%$ accuracy and F1 score improvements, respectively.

From the above experiments, we can conclude that SECRET leads to either significantly higher or comparable classification performance with respect to the ensemble method.

\subsection{RF Decision Node Depth}

In this section, we provide insight into how SECRET's semantic space RF models differ from the traditional feature space ones. Since SECRET uses meaning-based relationships among labels, it is able to divide the classes into 'easyto-classify' and 'difficult-to-classify'. We expect SECRET to adjust the RF decision node depths according to both the semantic relationships among labels and data characteristics, and traditional approaches to adjust only according to data characteristics. Therefore, we hypothesize that the decision node depth for different classes varies more in SECRET compared to the traditional approaches as labels show heterogeneous distribution in the semantic space and SECRET is able to integrate this information into the classification task (focus on 'difficult-to-classify' classes in deeper nodes with the help of its semantic space component).

We carry out the RF decision node depth experiments on six datasets (cmc, chess, lymph, cardio, nursery, and letter) to validate our hypothesis. The remaining four datasets (sonar, liver, wdbc, and heart) have two classes. When one class is assigned, the other class also gets distinguished. Therefore, the variance of the decision node depth for these four datasets tends to zero, which is not informative. For the six datasets that include three or more classes, we take each decision tree in the RF model and assess the decision nodes, their depth, and assigned classes. It is important to note that the RF models for the feature and semantic spaces are obtained with a classifier and a regressor, respectively.
To make a fair comparison, we convert the semantic-space RF regressor to a classifier by performing labeling with only the regressor outputs. For both models, within a tree, we compute the average decision node depth for each class. We repeat this process for each tree to assess the overall average decision node depth for the RF model.

As an example, Table 2 shows RF decision node depth variance and classification performance on the $\mathrm{cmc}$ dataset for both the traditional approaches and SECRET. While the traditional approaches assign 'no use,' 'long-term methods,' and 'short-term methods' at closer node depths by taking data characteristics into account, SECRET uses both the data characteristics and semantic relationships among labels (Fig. 2). As the 'no use' class is located farther away (in Euclidean distance) from the 'long-term methods,' and 'short-term methods' classes, SECRET assigns 'no use' to shallower depths and focuses on details to distinguish 'longterm methods,' and 'short-term methods' at deeper nodes. We summarize this result into one value, which is the overall variance of the RF node depth among all classes and 10 folds. While SECRET takes the heterogeneous distribution of the labels (in the semantic space) into account and shapes the tree depths accordingly, the traditional classifiers or ensembles ignore this point. They tend to assign labels at similar average node depths, resulting in a smaller overall variance compared to SECRET. As a result of SECRET's directed attention to 'easy-to-classify' and 'difficult-to-classify' classes, it outperforms the traditional approaches, as shown in the right column of Table 2 For the remaining datasets, we carried out the same analyses. Fig. 15 shows the overall variance of RF decision node depth for 'Traditional classifier,' 'Traditional ensemble,' and 'SECRET.' In Fig. 15a and Fig. 15b. 'Traditional classifier' represents the variance of RF model's decision node depth. In Fig. 15a. 'Traditional Ensemble' and 'SECRET' represent variance of decision node depth of RF models that are built on top of the MLP model, as shown in Fig. $4 \mathrm{~b}$ and Fig. 10, respectively. In Fig. 15b. 'Traditional Ensemble' and 'SECRET' are built on 
TABLE 2

Average RF Decision Node Depth, Overall RF Node Depth Variance and Classification Performance on the $\mathrm{cmc}$ Dataset

\begin{tabular}{|c|c|c|c|c|c|c|}
\hline \multirow[t]{2}{*}{ Approach } & \multicolumn{3}{|c|}{ Average RF Node Depth } & \multirow{2}{*}{$\begin{array}{l}\text { Overall Variance } \\
\text { of RF Node Depth }\end{array}$} & \multicolumn{2}{|c|}{ Classification Performance } \\
\hline & no use & long-term methods & short-term methods & & Accuracy (\%) & F1 Score $(\%)$ \\
\hline Traditional Classifier & 11.6 & 12.4 & 12.4 & 0.2 & 49.8 & 46.8 \\
\hline $\begin{array}{l}\text { Traditional Ensemble } \\
\text { (built on top of MLP) }\end{array}$ & 11.6 & 12.5 & 12.5 & 0.2 & 51.3 & 48.5 \\
\hline $\begin{array}{l}\text { Traditional Ensemble } \\
\text { (built on top of RF) }\end{array}$ & 11.5 & 12.4 & 12.4 & 0.2 & 50.6 & 47.9 \\
\hline $\begin{array}{l}\text { SECRET } \\
\text { (built on top of MLP) }\end{array}$ & 11.0 & 12.3 & 12.2 & 0.4 & 52.9 & 50.5 \\
\hline $\begin{array}{l}\text { SECRET } \\
\text { (built on top of RF) }\end{array}$ & 11.0 & 12.3 & 12.2 & 0.4 & 51.3 & 48.7 \\
\hline
\end{tabular}

top of an RF model. In five of the datasets (except lymph), we observe a larger variance in the overall decision node depth of SECRET compared to the traditional approaches. In line with this observation, SECRET obtains up to $11.2 \%$ and $12.1 \%$ accuracy and F1 score improvements, respectively, over the traditional classifier and up to $7.6 \%$ and $7.4 \%$ accuracy and F1 score improvement in accuracy and F1 score over the traditional ensemble method depicted in Fig. 10 For the other case shown in Fig. 15b, SECRET obtains up to $11.8 \%$ and $11.7 \%$ accuracy and F1 score improvements, respectively, over the traditional classifier and up to $11.9 \%$ and $11.8 \%$ improvements in accuracy and F1 score, respectively, over the traditional ensemble method. For the letter dataset, we observe comparable performance (maximum $0.3 \%$ decrease in accuracy/F1 score) with the traditional approaches. For the lymph dataset, while RF node depth variance is smaller for SECRET, we observe $0.1 \%$ to $3.6 \%$ accuracy and $0.0 \%$ to $5.8 \%$ improvement over the traditional approaches. This is inconclusive. As we also obtain inconclusive results throughout Section 5 due to its size, we do not discuss the lymph dataset further.

Overall, a larger variance in RF node depth indicates that SECRET is distinguishing 'easy-to-classify' and 'difficult-toclassify' cases more clearly than the traditional approaches and focusing on detailed characteristics at deeper nodes to separate the 'difficult-to-classify' cases further. As a result, we observe an enhancement in classification performance with SECRET. This is commensurate with our hypothesis.

\section{Related Work}

Next, we focus on related studies in both the feature and semantic spaces.

Enhancing classification performance of the ML algorithms has been a well-targeted area of research for decades. Various approaches have been proposed. These include data augmentation [23], data generation [24], boosting [25], ensemble learning [26], and dimensionality reduction [27]. In addition to these promising techniques, various ML algorithms (information-based, similarity-based, probabilitybased, and error-based [19|) and architectures have been designed. Specifically, for big data, neural network models [28 have revolutionized the classification task due to their ability to model complex data-label relationships. Although these algorithms and techniques have made significant contributions to enhancing classification performance, they all operate in the feature space. SECRET closes the gap between the feature and semantic spaces.

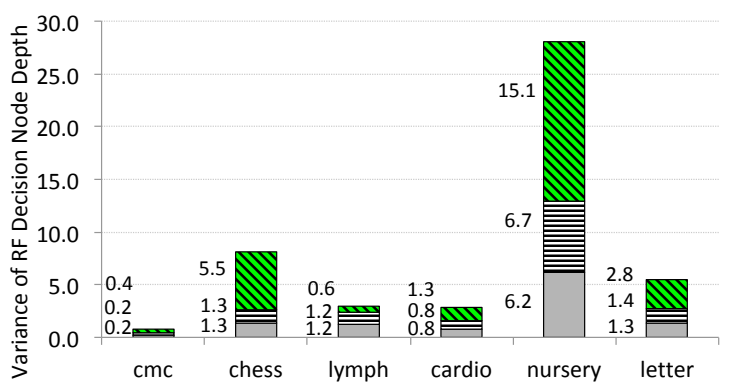

$\square$ Traditional classifier (Fig. 4a) $\mathbf{\theta}$ Traditional ensemble (Fig. 10) \$ SECRET (Fig.4b)

(a)

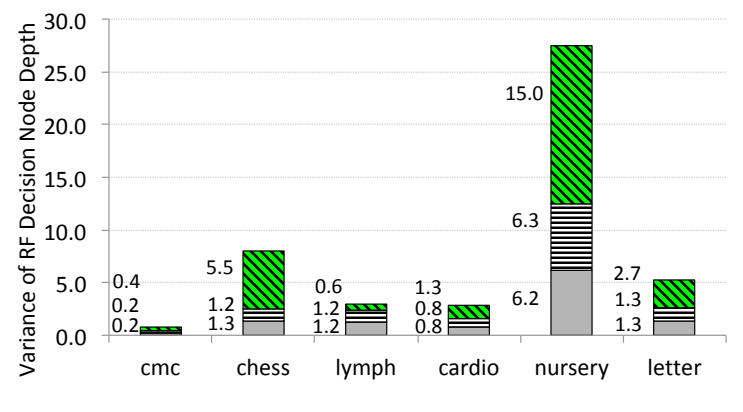

(b)

Fig. 15. Variance of RF decision node depth for traditional RF classifier and traditional ensemble and SECRET built on top of (a) MLP and (b) RF.

If we change our perspective and look at the related work in the semantic space, we observe that word representations have been widely used in NLP applications. Liu et al. [29] proposed a novel task-oriented word embedding method to assess the salient word for the text classification task. All analyses are carried out in the semantic space. Kusner et al. [30] introduced a novel distance metric (Word Mover's Distance) to effectively model the text documents with a set of word vectors. Vector representations act as features in a traditonal classification task and are mapped to a pre-defined set of labels with the k-Nearest Neighbor algorithm. This is a feature space approach since word vectors are used as features and mapped to a specific set of labels, without considering the meaning-based relationships among labels. Bordes et al. [31] targeted question answering by representing the question in a vector form in the semantic space and mapping it to the answer again in the semantic space. Bengio and Heigold [32] go far away from the semantic space by training vector representations of words without considering their meaning relationships, but target- 


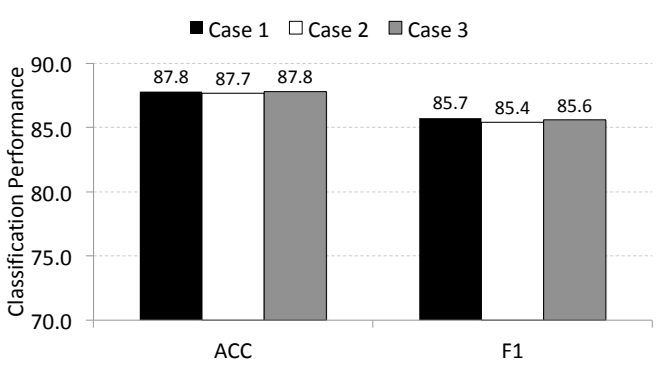

(a)

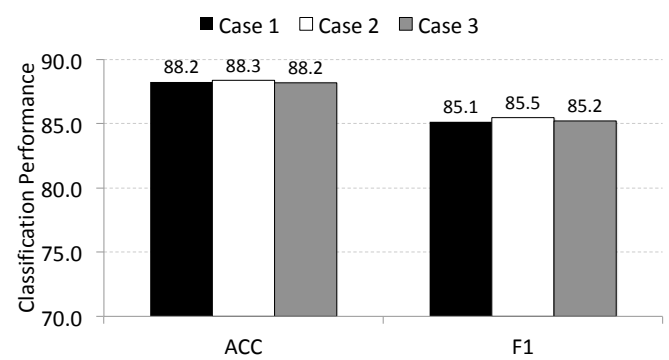

(b)

Fig. 16. Classification performance of SECRET on the cardio dataset when built with (a) MLP and (b) RF as the feature space classifier and $\mathrm{RF}$ as the semantic space regressor. Case 1 represents the performance of the cardio dataset when the labels are used as they are. Case 2 and Case 3 represent the performance when the 'REM sleep' label is replaced with 'paradoxical sleep' and 'dreaming sleep,' respectively.

ing how similar the words sound. Vectors of sound-alike (not semantically similar) words have a smaller Euclidean distance between them. Wang et al. |33| focused on the design of a CNN-RNN framework that maps image data to label embedding to perform multi-label classification while taking label co-occurrence and semantic redundancy into account. The proposed approach targeted only the image datasets with a fixed ML design. Palatucci et al. [2] and Socher et al. [34| carried out zero-shot learning by mapping real-world data to semantic vector representations of words. Karpathy and Fei-Fei [3] obtained figure captions using image datasets and word embeddings. The approaches presented in [2], [34], and [3] are limited to the semantic space. They only targeted correlations between data features and semantic relationships.

Overall, the above-mentioned approaches have had a significant influence on the development of NLP applications; however, they exploit either the feature space or the semantic space when performing classification. SECRET integrates these two spaces. Thus, SECRET can be differentiated from previous work and looks at real-world classification tasks in a new way.

\section{Discussion}

In this section, we analyze SECRET from different perspectives.

\subsection{What to do for classes with the same label, but different meanings?}

Word embedding algorithms, such as GloVe, capture the word's semantic relationships with the remaining words in the dictionary. Words with close meanings are represented by closely-spaced vectors in the semantic space. Therefore,

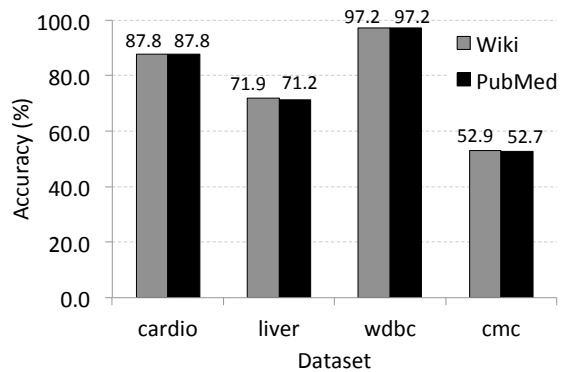

(a)

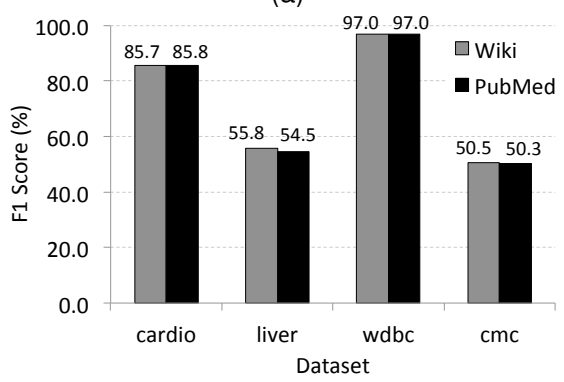

(b)

Fig. 17. Comparison of SECRET's (a) accuracy and (b) F1 score values when built with pretrained Wikipedia 2014 + Gigaword (Wiki) and PubMed word vectors. SECRET uses MLP as the feature space classifier and RF as the semantic space regressor.

vectors do not represent the sounding, but the meaning relationships between words. None of the datasets used in our experiments (Table 1) included homographs (words with the same spelling, but different meanings). However, in case of a homograph (such as 'spring'), the corresponding semantic vectors should be obtained with context-aware word embedding algorithms, as proposed by studies in [35], [36], [37], [38].

\subsection{How to decide on the semantic vector of a class whose label has synonyms?}

We hypothesize that SECRET provides very similar classification outputs when the synonyms of a class label are used interchangeably. We tested this hypothesis on the cardio dataset. As indicated by studies in [39] and [40], the 'REM sleep' label means the same as 'paradoxical sleep' and 'dreaming sleep.' Fig. 16 shows the classification accuracy and F1 scores for three cases when SECRET is built on top of the MLP and RF feature space classifiers. In all cases, SECRET obtains nearly the same classification performance, buttressing our hypothesis.

\subsection{Do the improvements come from the selected word embedding or SECRET? How does SECRET perform when a different word embedding is introduced?}

In order to demonstrate the effectiveness of SECRET and its performance stability on various word vectors, we repeated the experiments with a different set of pretrained word vectors |13| that is obtained using PubMed texts and the word2vec algorithm. PubMed stores biomedical literature and the corresponding word vectors are 200-dimensional. Considering the application area and size of the datasets (we need to avoid overfitting), we focus on the cardio, liver, wdbc, and cmc datasets. Fig. 17 shows the classification 


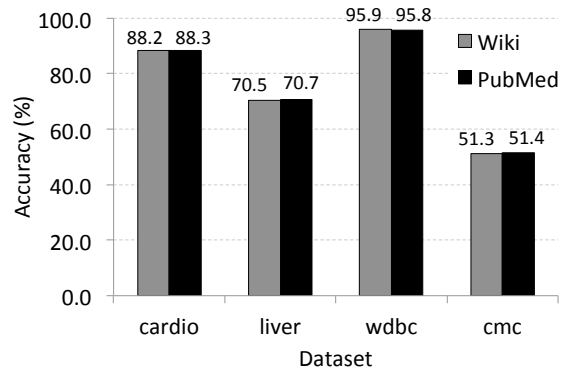

(a)

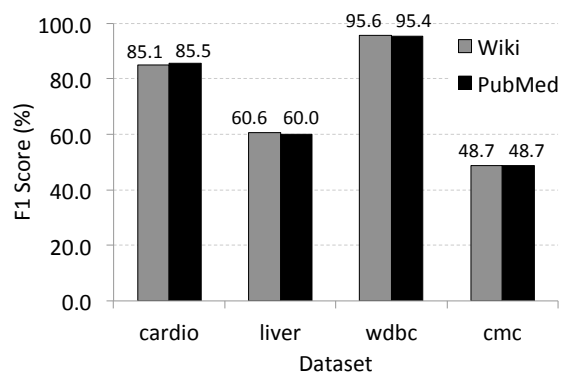

(b)

Fig. 18. Comparison of SECRET's (a) accuracy and (b) F1 score values when built with pretrained Wikipedia 2014 + Gigaword (Wiki) and PubMed word vectors. SECRET uses RF as the feature space classifier and $\mathrm{RF}$ as the semantic space regressor.

performance of SECRET when built with MLP as the feature space classifier and RF as the semantic space regressor. Similarly, Fig. 18 shows the performance with RF as the feature space classifier as well as the semantic space regressor. In both figures, we observe nearly identical classification performance for Wiki and PubMed-based word vectors. These results provide evidence that the classification performance improvements demonstrated in Figs. 8, 9, 11, 12, 13, and 14 do not arise from Wiki-based word vectors, but the integration of semantic information into the classification task.

\subsection{Does the semantic space regressor by itself per- form better than SECRET?}

We carried out additional experiments for the semantic space (only) case. We show the results in Fig. 19 and Fig. 20 for MLP and RF regressors, respectively. As in the case of the feature space (only), SECRET dominates the semantic space (only) too. This result was expected since SECRET integrates the feature and semantic spaces to benefit from their complementary advantages.

\section{Conclusion And Future Work}

In this article, we introduced a new dimension (semantic space) to the feature space based decision-making employed in ML algorithms and encapsulated it in a dual-space classification approach called SECRET. As opposed to traditional approaches, SECRET maps data to labels while integrating meaning-based relationships among labels. We analyzed SECRET's classification performance on ten datasets that represent different real-world applications. Among ten different real-world datasets, compared to traditional supervised learning, SECRET achieved up to $14.0 \%$ accuracy and $13.1 \%$
F1 score improvements. Compared to ensemble methods, SECRET achieved up to $12.7 \%$ accuracy and $13.3 \%$ F1 score improvements. We also took a step toward understanding how SECRET builds the semantic space component and its impact on overall classification performance. We posit that, in future work, further improvements in SECRET's overall classification performance and feature/semantic space characteristics can be made as follows. First, further analyses of different datasets are needed to support extensive applicability of SECRET. Second, although MLP and RF are well-known supervised ML algorithms, other ML algorithms need to be analyzed in this context. Third, semantic vectors could be trained specially for SECRET and the corresponding application of interest, as done in the case of intrinsic and extrinsic analyses in NLP [41], |42]. Fourth, detailed classification analyses need to be carried out for the multilabel classification task, where SECRET can be implemented with multilabel classification and regression algorithms targeted at the feature and semantic spaces, respectively. Finally, in addition to the feature and semantic spaces, other information sources for classification should be explored.

\section{REFERENCES}

[1] J. Hirschberg and C. D. Manning, "Advances in natural language processing," Science, vol. 349, no. 6245, pp. 261-266, 2015.

[2] M. Palatucci, D. Pomerleau, G. E. Hinton, and T. M. Mitchell, "Zero-shot learning with semantic output codes," in Proc. Advances in Neural Inf. Process. Syst., 2009, pp. 1410-1418.

[3] A. Karpathy and L. Fei-Fei, "Deep visual-semantic alignments for generating image descriptions," in Proc. IEEE Conf. Comput. Vision and Pattern Recognition, 2015, pp. 3128-3137.

[4] J. Bergstra, D. Yamins, and D. D. Cox, "Making a science of model search: Hyperparameter optimization in hundreds of dimensions for vision architectures," J. Machine Learning Research, vol. 28, 2013.

[5] B. Shahriari, K. Swersky, Z. Wang, R. P. Adams, and N. De Freitas, "Taking the human out of the loop: A review of Bayesian optimization," Proc. IEEE, vol. 104, no. 1, pp. 148-175, 2016.

[6] T. Mikolov, K. Chen, G. Corrado, and J. Dean, "Efficient estimation of word representations in vector space," arXiv preprint arXiv:1301.3781, 2013.

[7] J. Pennington, R. Socher, and C. Manning, "GloVe: Global vectors for word representation," in Proc. Conf. Empirical Methods in Natural Language Process., 2014, pp. 1532-1543.

[8] A. Mnih and K. Kavukcuoglu, "Learning word embeddings efficiently with noise-contrastive estimation," in Proc. Advances in Neural Inf. Process. Syst., 2013, pp. 2265-2273.

[9] R. Lebret and R. Collobert, "Word emdeddings through Hellinger PCA," arXiv preprint arXiv:1312.5542, 2013.

[10] T. Mikolov, M. Karafiát, L. Burget, J. Černockỳ, and S. Khudanpur, "Recurrent neural network based language model," in Proc. Eleventh Annual Conf. Int. Speech Commun. Association, 2010.

[11] "GloVe pre-trained word vectors," https://nlp.stanford.edu/ projects/glove/ accessed: 02-10-2019.

[12] "word2vec pre-trained word vectors," https://code.google.com/ archive/p/word2vec/. accessed: 02-10-2019.

[13] "word2vec pre-trained word vectors for biomedical applications," http://bio.nlplab.org accessed: 02-10-2019.

[14] M. C. Motwani, M. C. Gadiya, R. C. Motwani, and F. C. Harris, "Survey of image denoising techniques," in Proc. Int. Pervasive Signal Process. Conf. Exhibition, vol. 2004, 2004, pp. 27-30.

[15] S. L. Joshi, R. A. Vatti, and R. V. Tornekar, "A survey on ECG signal denoising techniques," in Proc. IEEE Int. Conf. Commun. Syst. Network Technologies, 2013, pp. 60-64.

[16] A. Kandaswamy, V. Krishnaveni, S. Jayaraman, N. Malmurugan, and K. Ramadoss, "Removal of ocular artifacts from EEG - A survey," Institution of Electron. Telecommunication Eng. J. Research, vol. 51, no. 2, pp. 121-130, 2005. 


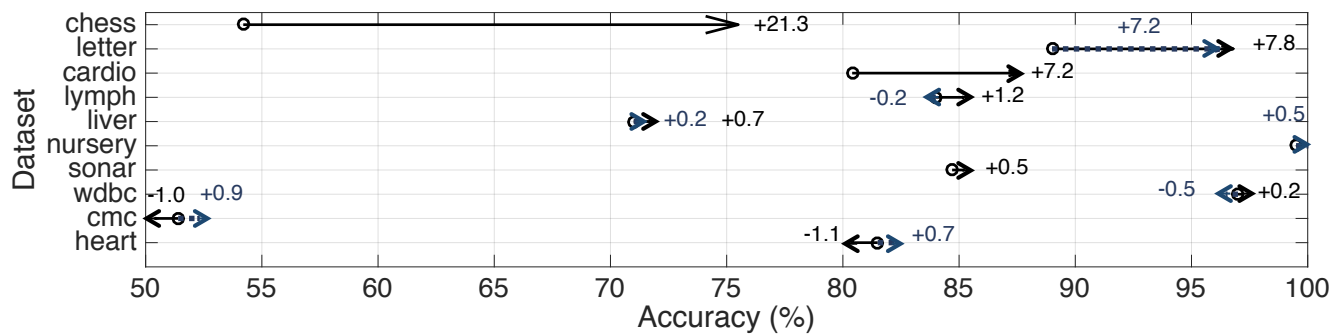

(a)

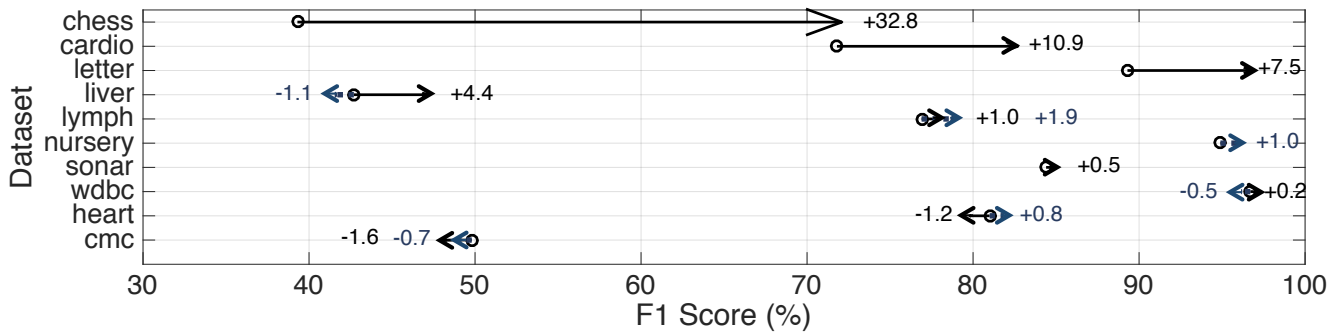

(b)

Fig. 19. SECRET's (a) accuracy and (b) F1 score improvements over the MLP regressor in the semantic space. SECRET uses MLP as the semantic space regressor and RF/MLP as the feature space classifier. Black arrows indicate when the RF classifier is used, whereas the dark blue and dashed arrows correspond to the MLP classifier.

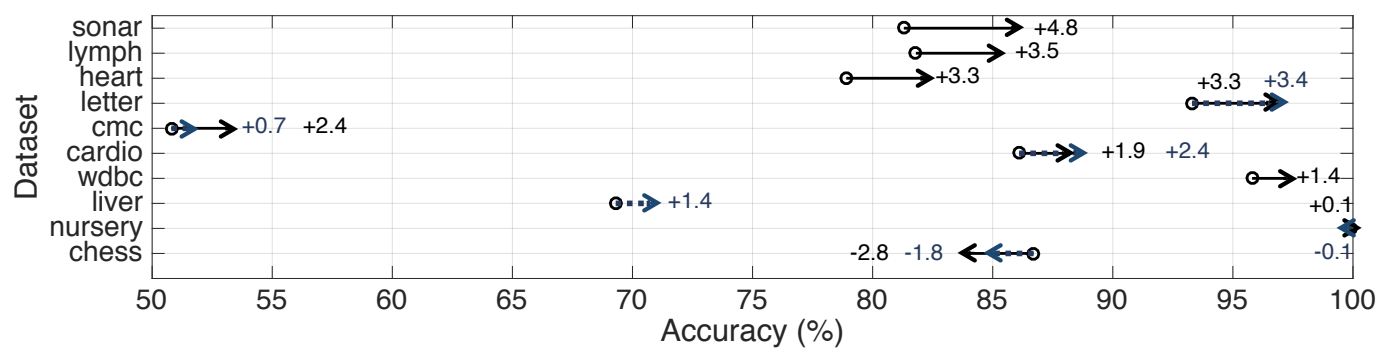

(a)

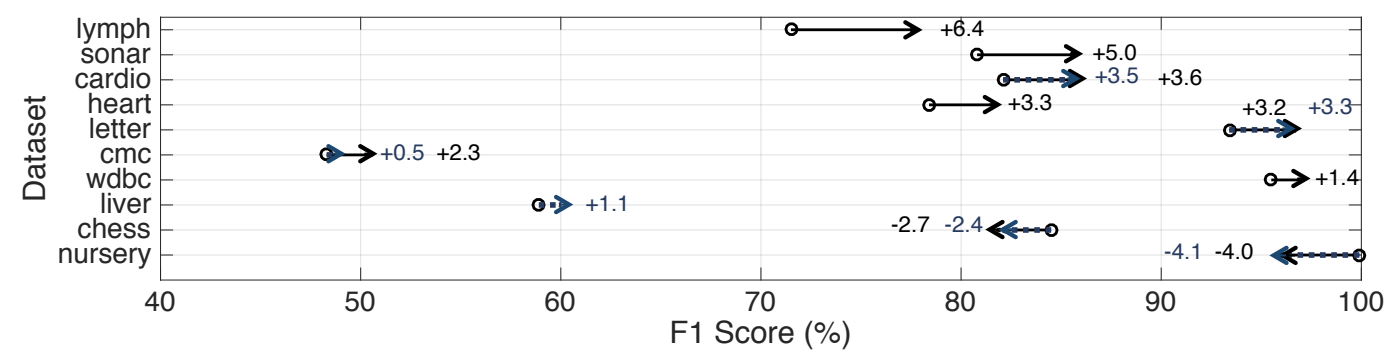

(b)

Fig. 20. SECRET's (a) accuracy and (b) F1 score improvements over the RF regressor in the semantic space. SECRET uses RF as the semantic space regressor and MLP/RF as the feature space classifier. Black arrows indicate when the MLP classifier is used, whereas the dark blue and dashed arrows correspond to the RF classifier.

[17] J. Mohan, V. Krishnaveni, and Y. Guo, "A survey on the magnetic resonance image denoising methods," Biomedical Signal Process. Control, vol. 9, pp. 56-69, 2014.

[18] V. Hodge and J. Austin, "A survey of outlier detection methodologies," Artificial Intelligence Review, vol. 22, no. 2, pp. 85-126, 2004.

[19] J. D. Kelleher, B. Mac Namee, and A. D'Arcy, Fundamentals of Machine Learning for Predictive Data Analytics. MIT Press, 2015.

[20] D. Dua and E. Karra Taniskidou, "UCI machine learning repository," 2017. [Online]. Available: http://archive.ics.uci.edu/ $\mathrm{ml}$

[21] “Bayesian optimization framework," https://github.com/fmfn/ BayesianOptimization accessed: 03-06-2019.

[22] F. Pedregosa, G. Varoquaux, A. Gramfort, V. Michel, B. Thirion, O. Grisel, M. Blondel, P. Prettenhofer, R. Weiss, V. Dubourg, J. Vanderplas, A. Passos, D. Cournapeau, M. Brucher, M. Perrot, and E. Duchesnay, "Scikit-learn: Machine learning in Python," J. Machine Learning Research, vol. 12, pp. 2825-2830, 2011.

[23] D. A. Van Dyk and X.-L. Meng, "The art of data augmentation," J. Computational and Graphical Statistics, vol. 10, no. 1, pp. 1-50, 2001.
[24] H. He, Y. Bai, E. A. Garcia, and S. Li, "ADASYN: Adaptive synthetic sampling approach for imbalanced learning," in Proc. IEEE Int. Joint Conf. on Neural Networks, 2008, pp. 1322-1328.

[25] Y. Freund, R. Schapire, and N. Abe, "A short introduction to boosting," Journal-Japanese Society For Artificial Intelligence, vol. 14 no. 771-780, p. 1612, 1999.

[26] T. G. Dietterich et al., "Ensemble learning," The Handbook of Brain Theory and Neural Networks, vol. 2, pp. 110-125, 2002.

[27] L. Van Der Maaten, E. Postma, and J. Van den Herik, "Dimensionality reduction: A comparative," J. Machine Learning Research, vol. 10, no. 66-71, p. 13, 2009.

[28] M. Z. Alom, T. M. Taha, C. Yakopcic, S. Westberg, P. Sidike, M. S. Nasrin, M. Hasan, B. C. Van Essen, A. A. Awwal, and V. K. Asari, "A state-of-the-art survey on deep learning theory and architectures," Electronics, vol. 8, no. 3, p. 292, 2019.

[29] Q. Liu, H. Huang, Y. Gao, X. Wei, Y. Tian, and L. Liu, "Taskoriented word embedding for text classification," in Proc. Int. Conf. Computational Linguistics, 2018, pp. 2023-2032.

[30] M. Kusner, Y. Sun, N. Kolkin, and K. Weinberger, "From word 
embeddings to document distances," in Proc. Int. Conf. Machine Learning, 2015, pp. 957-966.

[31] A. Bordes, J. Weston, and N. Usunier, "Open question answering with weakly supervised embedding models," in Proc. Joint European Conf. Machine Learning and Knowledge Discovery in Databases. Springer, 2014, pp. 165-180.

[32] S. Bengio and G. Heigold, "Word embeddings for speech recognition," in Proc. Fifteenth Annual Conf. Int. Speech Commun. Association, 2014, pp. 1053-1057.

[33] J. Wang, Y. Yang, J. Mao, Z. Huang, C. Huang, and W. Xu, “CNNRNN: A unified framework for multi-label image classification," in Proc. IEEE Conference on Computer Vision and Pattern Recognition, 2016, pp. 2285-2294.

[34] R. Socher, M. Ganjoo, C. D. Manning, and A. Ng, "Zero-shot learning through cross-modal transfer," in Proc. Advances in Neural Information Processing Syst., 2013, pp. 935-943.

[35] P. Liu, X. Qiu, and X. Huang, "Learning context-sensitive word embeddings with neural tensor skip-gram model," in Proc. TwentyFourth Int. Joint Conference on Artificial Intelligence, 2015.

[36] Y. Liu, Z. Liu, T.-S. Chua, and M. Sun, "Topical word embeddings," in Proc. AAAI Conf. Artificial Intelligence, 2015.

[37] D. Q. Nguyen, "An overview of embedding models of entities and relationships for knowledge base completion," arXiv preprint arXiv:1703.08098, 2017.

[38] G.-H. Lee and Y.-N. Chen, "MUSE: Modularizing unsupervised sense embeddings," arXiv preprint arXiv:1704.04601, 2017.

[39] H. H. Jasper and J. Tessier, "Acetylcholine liberation from cerebral cortex during paradoxical (rem) sleep," Science, vol. 172, no. 3983, pp. 601-602, 1971.

[40] R. Greenberg, R. Pillard, and C. Pearlman, "The effect of dream (stage REM) deprivation on adaptation to stress." Psychosomatic Medicine, 1972.

[41] P. Resnik and J. Lin, "Evaluation of NLP systems," The Handbook of Computational Linguistics and Natural Language Processing, vol. 57, pp. 271-295, 2010.

[42] M. Zhai, J. Tan, and J. D. Choi, "Intrinsic and extrinsic evaluations of word embeddings." in Proc. Association for the Advancement of Artificial Intell., 2016, pp. 4282-4283. 